\title{
De nouveaux seconds messagers de l'action hormonale
}

De découverte récente, les produits de dégradation du phosphatidyl-inositol se révèlent être les seconds messagers de nombreuses hormones et probablement, de facteurs de croissance. Ils jouent vraisemblablement un rôle pivot dans la régulation de la prolifération cellulaire.

\section{Christian Le Peuch}

Chargé de recherches à l'Institut national de la santé et de la recherche médicale. U249 de l'Inserm et LP 8402 du Cnrs.

\section{REFERENCES}

1. Berridge $\mathbf{M} \mathrm{J}$. Inositol trisphosphate and diacylglycerol as second messengers. Biochem 7 $1984 ; 220: 345-60$.

2. Berridge $M$ J, Irvine $R$ F. Inositol trisphosphate, a novel second messenger in cellular signal transduction. Nature $1984 ; 312: 315-21$.

\section{ADRESSE}

Ch. L.c Pcuch, U 249 de l'Inscrm et L.P $8_{4} 02$ du Cnrs. BP 5051, 34033 Montpellier. 'évolution des organismes pluricellulaires repose sur la capacité des cellules à communiquer entre elles. Les communications d'une extrême complexité chez les organismes supérieurs sont nécessaires au contrôle du développement des cellules, de leur organisation en tissus ou organes, de leur croissance et de leur multiplication, nécessaires aussi à la coordination de leurs activités. Dans l'un des systèmes de communication, un premier signal appelé premier messager (hormone, facteur de croissance...) est secrété et circule dans le milieu extracellulaire. Le premier messager est " capté " à la surface des cellules par des récepteurs qui lui sont spécifiques. L'occupation des récepteurs déclenche des événements complexes, à la fois dans la membrane plasmique et à l'intérieur de la cellule. Le premier signal est simultanément amplifié et traduit en un second signal " porté " par un second messager intracellulaire c'est la transduction du signal hormonal. La découverte de l'accroissement de la synthèse et de la concentration d'adénosine monophosphate cyclique (AMPc) dans une cellule stimulée par une hormone est à l'origine du concept de second et premier messagers Depuis lors, les ions calcium $\mathrm{Ca}^{2}+$ et plus récemment encore, le sn1,2-diacylglycerol et l'inositol1,4,5-trisphosphate, tous deux produits de dégradation du phosphatidylinositol-4,5-bisphosphate, ont été identifiés comme des seconds messagers. La compréhension des mécanismes qui conduisent de la réception du premier messager à la réponse biologique de la cellule est fondamentale, d'autant qu'il semble que des dérèglements dans la transduction par la cellule des signaux hormonaux soient à l'origine de nombreuses maladies ainsi que de la transformation cellulaire qui peut conduire au cancer.

\section{Métabolisme des phosphoinositides}

Les principales étapes du cycle de l'inositol et des phosphoinositides sont connues et ont fait l'objet de publications récentes $[1,2]$. Ce cycle peut être résumé comme suit : l'inositol intracellulaire provient du 
RÉFÉRENCES
3. Hokin M R, Hokin L E. Enzyme secretion and
the incorporation of ${ }^{32} \mathrm{P}$ into phospholipids of
pancreas slices. 7 Biol. Chem $1953 ; 203: 967-77$.
4. Durrel J, Garland $\mathrm{J} T$, Friedel $\mathrm{R}$ O. Ace-
tylcholine action: Biochemical aspects. Two major
approaches to understanding the mechanism of
action of acetylcholine are examined. Science
1969; $165: 862-6$.
5 . Schacht J, Agranoff B W. Effects of acetylcho-
line on labeling of phosphatidate and phosphoino-
sitides by 32P orthophosphate in nerve ending
fractions of guinea pig cortex. 7 Biol Chem 1972;
$247: 771-7$.

6. Michell $\mathrm{R}$ H. Inositol phospholipids and cell surface receptor function. Biochim Biophys Acta $1975 ; 415: 81-147$.

7. Berridge $M \mathrm{~J}$. Rapid accumulation of inositol trisphosphate reveals that agonists hydrolyse polyphosphoinositides instead of phosphatidylinositol. Biochem 7 1983; 212 : 849-68.

8. Rodbell $M$. The role of hormone receptors and GTP-regulatory proteins in membrane transduction. Nature 1980; $284: 17-22$.

9. Gilman A. G-proteins and dual control of adenylate cyclase. Cell 1984; $36: 577-9$.

10. Haslam R J, Davison M M L. Guanine nucleotides decrease the free $\mathrm{Ca}^{2+}$ required for secretion of serotonin from permeabilized blood platelets. Evidence of a role for a GTP-binding protein in platelet activation. FEBS Lett 1984; $174: 90-5$.

11. Cockcroft S, Gomperts B D. Role of guanine nucleotide binding protein in the activation of polyphosphoinositide phosphodiesterase. Nature $1985 ; 314: 534-6$.

12. Streb H, Irvine R F, Berridge M J, Schulz I. Release of $\mathrm{Ca}^{2+}$ from a non mitochondrial intracellular store in pancreatic acinar cells by inositol-1,4,5-trisphosphate. Nature 1984; 306 : 67-9.

13. Whitaker $\mathbf{M}$, Irvine $\mathbf{R} \quad \mathrm{F}$. Inositol-1, 4,5-trisphosphate microinjection activates sea urchin eggs. Nature 1984; $312: 636-9$.

14. Picard A, Giraud F, Le Bouffant F, Sladeczek $F$, Le Peuch C, Dorée $M$. Inositol-1,4,5-trisphosphate microinjection triggers activation, but not meiotic maturation in amphibian and starfish oocytes. FEBS Lett 1985 ; $182: 446-50$.

15. Nishizuka $Y$. The role of protein kinase $C$ in cell surface signal transduction and tumour pro-

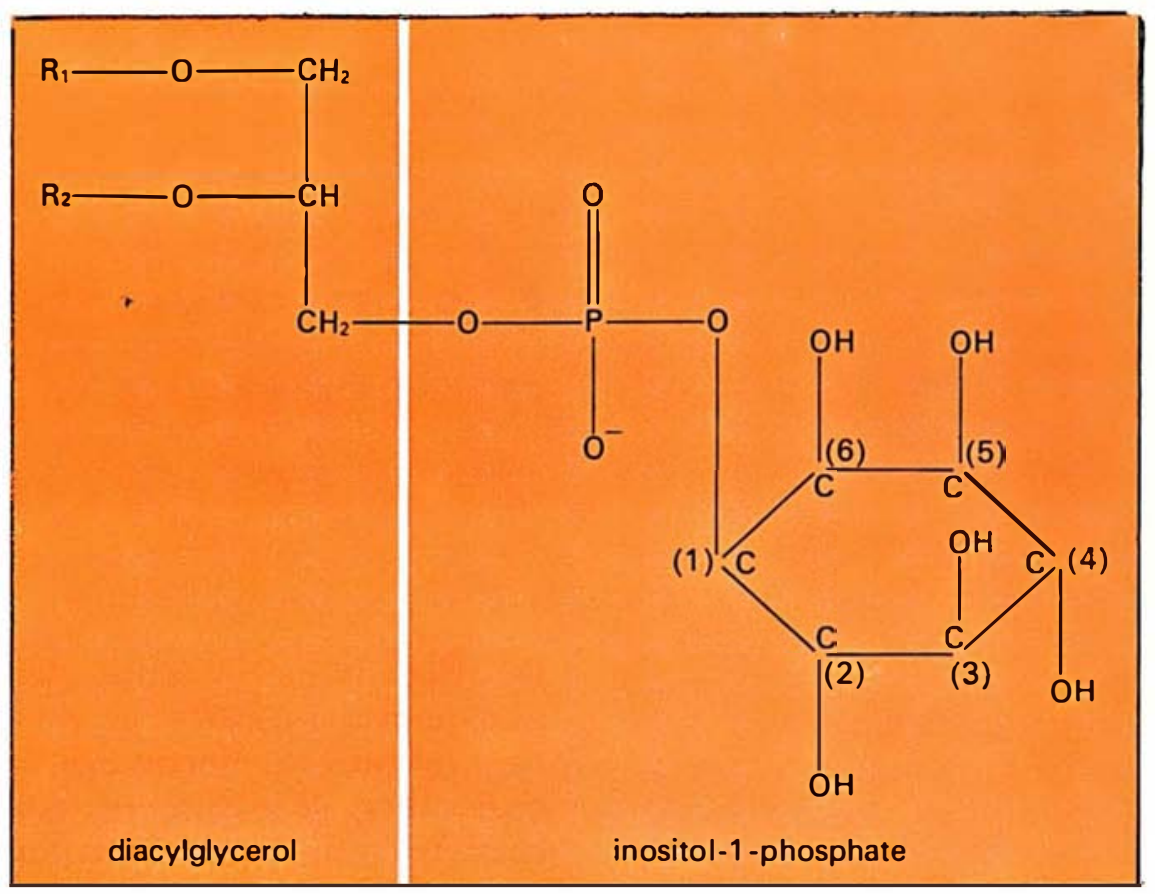

Figure I. Structure possible du phosphatidylinositol.

$R_{I}$ et $R_{2}$ sont des acides gras.

plasma ou de la déphosphorylation de l'inositol-1-phosphate par une phosphatase. Le phosphatidylinositol (PI) (figure ,) est formé à partir de l'inositol par réaction de ce dernier avec le cyrtidine diphosphate diacylglycerol (CDP-DG). Le PI formé est alors phosphorylé par une (ou plusieurs) kinase(s) en phosphatidylinositol-4-phosphate (PIP), puis en phosphatidylinositol-4,5-bisphosphate (PIP2). Ces deux dérivés phosphorylés du phosphatidylinositol peuvent être hydrolysés par des phosphodiestérases spécifiques, en sn-1,2-diacylglycerol (DG) d'une part, et inositol-1,4-bisphosphate (IP2), ou inositol-1,4,5-trisphosphate (IP 3 ) d'autre part (figure 2).

$\mathrm{L'IP}_{3}$ et le DG sont considérés comme les deux seconds messagers produits par la dégradation de PIP 2 par une phosphodiestérase stimulée lors de l'occupation de récepteurs membranaires. Comme pour tout second messager, l'élévation de la concentration de DG et d'IP 3 doit être transitoire afin de permettre un retour rapide de la cellule à " l'état de repos ». Ces conditions sont réalisées grâce à une kinase très active qui transforme le DG en acide phosphatidique, et à des phosphatases qui transforment $\mathrm{IP}_{3}$ en $\mathrm{IP}_{2}$, puis en IP (inositol-1-phosphate) et enfin en inositol. La phosphatase qui transforme IP en inositol est fortement inhibée par les ions lithium $\left(\mathrm{Li}^{+}\right)$. Cette propriété pourrait expliquer les effets du lithium sur certaines fonctions, en particulier au niveau du cerveau. Ceci est d'autant plus vrai que les membranes plasmiques des cellules du système nerveux sont imperméables à l'inositol plasmatique. Ainsi, dans ces cellules, les ions lithium bloqueront complètement le cycle de l'inositol et des phosphoinositides. Les enzymes responsables des différentes étapes de ce cycle ont des fonctions bien définies, mais on connaît mal leurs caractéristiques biochimiques et physicochimiques. En plus de son rôle de second messager, le sn-1,2-diacylglycerol est une source d'acide arachidonique, 
précurseur de métabolites tels que les prostaglandines, les leucotriènes, les tromboxanes et prostacyclines...

\section{Récepteurs et forma- tion de IP3 et DG}

La première observation sur le contrôle hormonal du métabolisme des phosphoinositides date de plus de 30 années. C'est en effet en 1953 que Hokin et Hokin [3] montrent que la stimulation du pancréas par l'acetylcholine accroît l'incorporation de ${ }^{32} \mathrm{P}$ dans les phospholipides. Il apparut par la suite qu'une grande variété d'agonistes stimulait le métabolisme des phospholipides membranaires et plus spécifiquement celui du phosphatidylinositol et de ses dérivés. Cependant, la première indication qu'une hydrolyse des phosphoinositides pouvait être induite en réponse à l'occupation de récepteurs par des agonistes fût obtenue par Durell [4]. Quelques années plus tard on montrait que l'acétylcholine réduisait le niveau de marquage des phosphatidylinositol-4 et $-4,5$-phosphates par ${ }^{32} \mathrm{P}$ [5]. Cette diminution du marquage des phospholipides membranaires coïncidait avec une augmentation de la concentration dans le cytosol d'IP 3 hydrosoluble. Dès 1975, Michell émettait l'hypothèse que la stimulation du métabolisme des inositides devait constituer une étape importante dans le mode d'action des agonistes mobilisateurs de calcium [6]. La liste des agonistes modifiant à la fois le métabolisme des phospholipides et la concentration du calcium intracellulaire s'allongeant, l'hypothèse de Michell semblait se confirmer. Cependant, deux problèmes subsistaient : d'une part, un chaînon manquait entre phosphoinositides et calcium; d'autre part, la modifica-

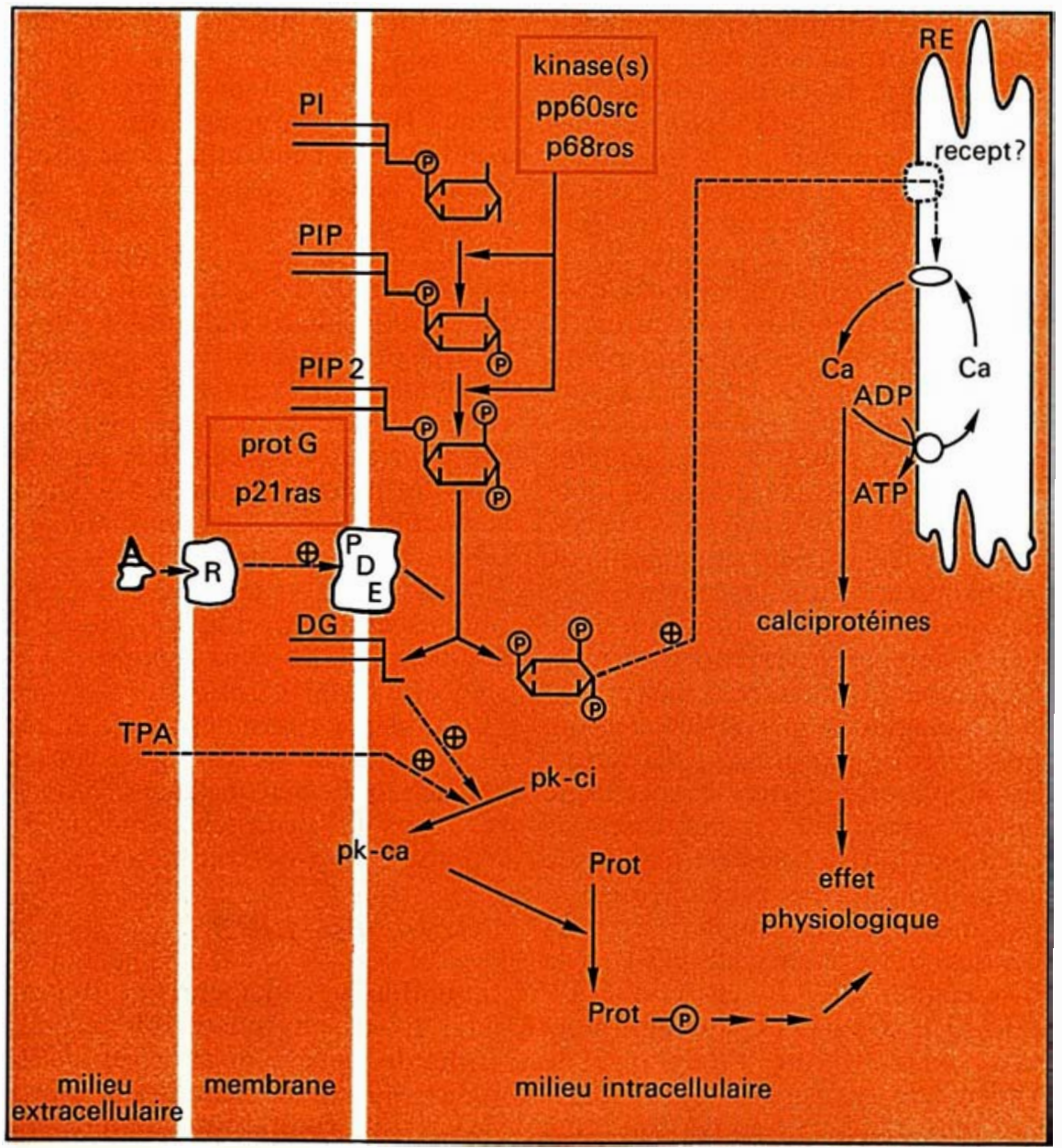

Figure 2. Biosynthèse et mécanisme d'action du sn-1,2-diacylglycérol et de l'inositol-I,4,5-trisphosphate.

Lorsqu'un agoniste hormonal $(A)$, se lie à son récepteur $(R)$, il $y$ a stimulation de la phosphodiestérase (PDE) qui hydrolyse le phosphatidylinositol-4,5bisphosphate en diacylglycérol (DG) et en inositol-1,4,5-trisphosphate (IP 3$)$. Une GTP-protéine (prot-G) intervient probablement dans le couplage entre $R$ et $P D E$. En présence de $D G$, la protéine kinase $C$ inactive (pk-Ci) et cytosoluble, se lie aux membranes, est activée (pkCa) et phosphoryle protéines et enzymes, dont l'activité se trouve modifiée et conduit à l'effet physiologique final. $I P_{3}$ se lie - à un récepteur? - au niveau du réticulum endoplasmique ( $R E)$ et stimule l'efflux de calcium. Certains produitsd'oncogènes (désignés en rouge) peuvent avoir la même activité que des enzymes responsables de certaines étapes et présentés dans un même cadre. 


\section{RÉFĖRENCES}

16. Takai $Y$, Kihkawi $K$, Nishizuka $Y$. Membrane phospholipid metabolism and signal transduction for protein phosphorylation. In: Greengard P, Robison G A, eds. Advances in Cycl Nucl Prot Phosph Res. New-York: Raven Presse, $1984 ; 18: 119-58$.

17. Castagna M, Takai Y, Kaibuchi K, Sano K, Kikkawa V, Nishizuka Y. Direct activation of calcium-activated, phospholipid-dependent protein kinase by tumor-promoting phorbol ester. 7 Biol Chem 1982; 257 : 7847-51.

18. Hinter T, Ling N, Cooper J A. Protein kinase C phosphorylation of the EGF receptor at a threonine residue close to the cytoplasmic face of the plasma membrane. Nature $1984 ; 311: 480-3$.

19. Moolenaar W H, Tertoolen L G J, Delaat S W. Phorbol ester and diacylglycerol mimic growth factors in raising cytoplasmic $\mathrm{pH}$. Nature $1984 ; 312: 371-4$.

20. Sugimoto Y, Whitman M, Cantley L C, Erikson R L. Evidence that the Rous sarcoma virus transforming gene product phosphorylates phosphatidylinositol and diacylglycerol. Proc Natl Acad Sci USA 1984; 82 : 2117-21.

21. Macara I G, Marinetti G V, Balduzzi P C. Transforming protein of avian sarcoma virus UR2 is associated with phosphatidylinositol kinase activity: possible role in tumorigenesis. Proc Natl Acad Sci USA 1984; 81 : 2728-32.

22. Shih T Y, Papageorge A G, Stokes P E, Weeks M O, Scolnick E M. Guanine nucleotidebinding and autophosphorylating activities associated with the $\mathbf{p} 21^{\text {sre }}$ protein of Harvey murine sarcoma virus. Nature 1980; $287: 686-91$.

23. Mc Grath J P, Capon D J, Goeddel D V, Levinson A D. Comparative biochemical properties of normal and activated human ras p21 protein. Nature 1984; 310:644.

24. Toda $T$, Uno I, Ishikawa $T$, et al. In yeast, RAS proteins are controlling elements of adenylate cyclase. Cell $1985 ; 40: 27-36$.

25. Burn P, Rotman A, Meyer R K, Burger M M. Diacylglycerol in large alpha-actinin/actin complexes and in the cytoskeleton of activated platelets. Nature $1985 ; 314: 469-72$.

26. Lassing I, Linderg V. Specific interaction between phosphatidylinositol-4,5-bisphosphate tion du métabolisme des phosphoinositides pouvait n'être que la conséquence de l'augmentation de la concentration de calcium intracellulaire due à l'ouverture de canaux à la suite de la liaison des agonistes à leur récepteur.

Aussi, l'observation que la formation de l'IP $_{3}$ dans des hépatocytes stimulés par la vasopressine était indépendante de la concentration de calcium extracellulaire fût-elle déterminante. A ceci devait s'ajouter la démonstration que dans les glandes salivaires de Calliphora (insecte) stimulées par la sérotonine, la formation $\mathrm{d}^{\prime} \mathrm{IP}_{3}$ précédait la formation d'autres phosphoinositides (IP, IP 2 ) et précédait la mobilisation du calcium intracellulaire [7]. Les mêmes observations devaient être faites sur le foie et sur des cellules $\mathrm{GH}_{3}$. Ainsi se confirmait l'idée que l'IP 3 était le chaînon manquant, le second messager responsable de la mobilisation du calcium intracellulaire.

Ces diverses observations montraient que la stimulation de la phosphatidylinositol-4,5-bis-phosphate phosphodiestérase, enzyme responsable de la dégradation du $\mathrm{PIP}_{2}$ en $\mathrm{IP}_{3}$ et DG, était l'événement fondamental de la transduction. Restait à préciser le mécanisme du couplage entre récepteurs et phosphodiestérase.

Le couplage entre récepteurs hormonaux et adénylate cyclase est le mieux connu. Schématiquement, lorsque l'hormone se lie à son récepteur, celui-ci interagit, probablement à la suite de changements de conformation, avec la sous-unité régulatrice de l'adénylate cyclase (Gs). Gs fait partie d'une famille de protéines appelées protéines- $G$ qui lient le GTP, possèdent une activité GTPasique et interviennent dans la régulation de nombreux phénomènes biologiques, parmi lesquels on peut citer l'activation dans la rétine, de la phosphodiestérase spécifique de la guanosine monophosphate cyclique (GMPc). Gs est formée de sous-unités, $\alpha, \beta$ et probablement $\gamma$, et l'interaction du récepteur avec Gs conduit, en présence de GTP, à la dissociation de Gs en Gs $\alpha-G T P$ plus Gs $\beta$. Gs $\alpha-G T P$ interagit alors avec la sous-unité catalytique de l'adénylate cyclase et l'active. L'hydrolyse du GTP lié à Gs $\alpha$ conduit à un retour du système à l'état de repos. Ceci explique pourquoi l'utilisation d'analogues non hydrolysables du GTP entraîne une activation permanente de l'adénylate cyclase. Les premières indications concernant l'intervention d'une protéine-G dans la transduction de signaux, conduisant à la mobilisation du calcium, sont venues de l'observation de la réduction par le GTP, des affinités de la noradrénaline pour les récepteurs alpha-1 et du carbachol pour les récepteurs de type muscarinique. Plus convaincants encore furent les résultats montrant que l'addition de GTP (ou de ses analogues non hydrolysables, le GTP- $\gamma \mathrm{S}$ ou le GppNHp) conduisait, dans des plaquettes perméabilisées, à un accroissement de la sensibilité au calcium des processus de sécrétion [10]. Les mêmes effets stimulateurs du GTP ou de ses analogues ont été observés dans d'autres systèmes cellulaires, en particulier dans les mastocytes où ils stimulent l'exocytose et la sécrétion d'histamine. Plus récemment, ces résultats ont été précisés [11] et l'intervention d'une protéine-G dans la transduction du signal conduisant à l'activation de la phosphodiestérase paraît donc se confirmer.

Ainsi, certains des mécanismes de la régulation de la phosphodiestérase du phosphatidylinositol-4,5-bisphosphate semblent se préciser. Cependant la caractérisation, en termes moléculaires, des protéines qui jouent un rôle régulateur dans la voie des phosphoinositides - kinases, phosphatases, phosphodiestérases... - débute à peine.

L'hydrolyse de $\mathrm{PIP}_{2}$ par une phosphodiestérase spécifique conduit à la libération de deux composés, le sn-1,2-diacylglycérol

l'inositol-1,4,5-trisphosphate. Il est donc important de distinguer la part respective de chacun de ces composés dans le déclenchement de la réponse biologique finale.

\section{Rôle de I'IP3}

Il semble actuellement que l'inositol-1,4,5-trisphosphate soit responsable de la mobilisation et de la redistribution du calcium à partir de certains compartiments intracel- 
lulaires où il est séquestré. Les premières suggestions de ce rôle viennent des études rapportées plus haut. Plus récemment, des expériences réalisées sur des cellules perméabilisées de pancréas [12], de foie ou de muscle lisse, indiquent sans ambiguïté que le calcium est relâché après addition d'IP 3 . Les réservoirs de calcium intracellulaire concernés n'ont pas toujours été clairement identifiés. Les résultats obtenus vont cependant dans le sens d'une action de l'IP $_{3}$ sur le réticulum endoplasmique. En effet, l'inhibition des fonctions mitochondriales, par suppression de substrats métaboliques ou par l'addition d'inhibiteurs, n'a aucun effet sur la sensibilité à l'IP 3 de la libération des ions calcium. Cette conclusion est confortée par des expériences réalisées sur des vésicules membranaires isolées d'insulinomes de cellules hépatiques ou cardiaques, ou enfin de plaquettes humaines. $\mathrm{L}^{\prime} \mathrm{IP}_{3}$ provoque la libération de calcium par ces vésicules membranaires dont la caractérisation, par des marqueurs enzymatiques, montre qu'il s'agit de microsomes formés à partir du réticulum endoplasmique lisse. Des expériences très récentes montrent que la microinjection d'IP 3 dans des ovocytes intactes, non perméabilisés, provoque la libération de calcium dans le cytoplasme et conduit à l'activation parthénogénétique des ovocytes $[13,14]$. Le fait que ces réactions ne soient pas observées lorsque les ovocytes ne possèdent pas de reticulum endoplasmique cortical différencié, confirme que ce système est la cible de l'IP 3 [14].

Comment le calcium est-il relâché par le réticulum endoplasmique? On suppose actuellement que des récepteurs spécifiques existent au niveau du réticulum endoplasmique. La liaison de l'IP 3 à ces récepteurs semble favoriser l'efflux de calcium plutôt qu'inhiber son pompage. En effet, lorsque le pompage du calcium par les ATPases du réticulum est complètement inhibé par l'addition d'ions vanadate, ou par élimination de l'ATP, on observe un efflux modéré des ions calcium. Cet efflux est en tout état de cause très inférieur à celui qu'on observe après addition d'IP ${ }_{3}$. Les drogues anticalciques qui bloquent le pas- sage des ions calcium au travers des canaux calciques sont sans effet sur la libération de calcium induite par l'IP 3 . Deux autres phosphoinositides, bien que moins efficaces que l'IP $_{3}$, peuvent également provoquer la libération d'ions calcium. Il s'agit de l'inositol-2,4,5-trisphosphate et de l'inositol-4,5-bisphosphate. Il semble que la présence d'une paire de phosphates vicinaux en position 4 et 5 soit indispensable à l'effet biologique, le phosphate en position 1 étant responsable de l'affinité élevée de $\mathrm{l'IP}_{3}$ pour son " récepteur".

\section{Rôle du DG ou sn-1,2-diacylglycerol}

Le sn-1,2-diacylglycerol est le second produit formé lors de l'hydrolyse du phosphatidylinositol-4,5bisphosphate. Toutefois ce composé peut aussi être produit par l'hydrolyse de n'importe quel phospholipide par une phosphodiestérase. Par son mode d'action, le DG rappelle l'AMPc. En effet, l'un des effets bien décrits du DG est l'activation d'une protéine kinase, la protéine kinase $\mathrm{C}$, en présence des ions calcium et de phospholipides. Les études concernant la protéine kinase C d'une part, et le métabolisme des phosphoinositides d'autre part, se sont développées relativement indépendamment jusqu'à une date très récente. Les pièces du puzzle sont venues s'ajuster pour renforcer encore l'importance de cette nouvelle voie de régulation.

La protéine kinase $\mathrm{C}$ a fait l'objet de nombreuses revues $[15,16]$. Elle fut découverte par le prof esseur Nishizuka et son équipe. En résumé, cette kinase est monomérique, elle a un poids moléculaire apparent d'environ 80000 , estimé aussi bien par électrophorèse en présence de détergent que par filtration sur gel. Cette kinase est activée in vitro par des phospholipides et plus spécifiquement par la phosphatidylsérine. L'activation nécessite, d'autre part, la présence de concentrations micromolaires d'ions calcium. Il semble qu'in vivo, dans une cellule non stimulée, la protéine kinase $\mathrm{C}$ soit présente dans le cytosol sous forme soluble. Lorsque la cellule est stimulée et que du DG est formé, il y a probablement translocation vers les membranes et activation de la kinase. L'intérêt pour la protéine kinase $C$ se trouva relancé voici quelques années par la découverte qu'un phorbol ester, le TPA (1 2-O-tetradecanoyl-phor-bol-1 3-acetate) était capable de se substituer au DG pour activer la kinase [17]. Le TPA est un promoteur de tumeurs, il facilite la transformation cellulaire et l'action des carcinogènes. De nombreux autres esters de phorbol ont été testés pour leur capacité à activer la protéine kinase C et - fait troublant - leur capacité à activer la protéine kinase $C$ est très bien corrélée avec leur pouvoir promoteur de tumeurs. On peut aussi ajouter que la protéine kinase $C$ est le seul récepteur protéique connu du TPA. On peut ici se poser la question suivante : pourquoi, si les actions du DG et du TPA sont finalement les mêmes, le DG n'estil pas promoteur de tumeurs? La réponse comporte deux raisons essentielles : d'abord contrairement au TPA qui n'est pas métabolisé, le DG est très rapidement transformé en acide phosphatidique, ensuite l'affinité du TPA pour la protéine kinase $\mathrm{C}$ est très supérieure à celle du DG.

La protéine kinase $C$ est activée par les ions calcium sans faire intervenir la calmoduline. C'est, à notre connaissance, la seule kinase présentant ces caractéristiques. Cependant, la protéine kinase $C$ est inhibée par des composés comme la fluphénazine et les naphtalènes sulfonamides qui sont également des inhibiteurs de l'action de la calmoduline. Ceci a amené certains auteurs à postuler l'existence dans la protéine kinase $\mathrm{C}$ d'un domaine identique à la calmoduline.

La protéine kinase $C$ est présente dans pratiquement tous les tissus qui ont été testés à ce jour. Sa concentration dans certains tissus comme le cerveau ou les plaquettes sanguines est très élevée. Malgré les données accumulées sur cette kinase, sa fonction exacte reste dans la plupart des cas un mystère. On ne dispose actuellement que de très peu d'exemples de phénomènes biologiques sur lesquels la protéine kinase $C$ ait un effet parfaitement démontré. La plupart des effets rap- 
portés comme étant la conséquence de l'activation de la protéine kinase ont été obtenus en traitant des cellules ou des tissus par le TPA, et en supposant donc que l'activation de la kinase est une étape obligatoire de l'action du TPA.

Le mécanisme d'action de la protéine kinase $\mathrm{C}$ a été bien étudié dans les cellules de carcinome humain A431. La membrane plasmique de ces cellules est riche en récepteurs pour un facteur de croissance, l'EGF (Epidermal Gromth Factor). La multiplication et la croissance des cellules $\mathrm{A}_{431}$ sont stimulées par l'addition d'EGF au milieu de culture. Si du TPA est également ajouté, il annule l'effet de l'EGF. De nombreuses expériences ont montré que l'effet du TPA pouvait s'expliquer selon le schéma suivant : le TPA active la protéine kinase $C$ qui phosphoryle alors le récepteur de l'EGF, cette phosphorylation conduit à une perte d'affinité du récepteur pour l'EGF qui devient ainsi inefficace [18]. A ce premier effet s'en ajoute un second : un des phénomènes biologiques induits par l'addition d'EGF aux cellules $\mathrm{A}_{43} 1$ est une élévation du $\mathrm{pH}$ intracellulaire, probablement via une modification des propriétés de l'échangeur $\mathrm{Na}^{+} / \mathrm{H}^{+}$de la membrane plasmique [19]. Cette élévation de $\mathrm{pH}$ serait elle-même responsable de divers événements et en particulier d'une reprise de la synthèse $d$ 'ADN. Le traitement par TPA des cellules $A_{43} 1$ induirait un bloquage au niveau de l'échangeur, dû à la phosphorylation - non encore démontrée - de l'échangeur par la kinase qui conduirait à un changement de ses propriétés. Des modifications du $\mathrm{pH}$ intracellulaire à la suite de traitement par le TPA ont été observées sur d'autres systèmes. L'ensemble de ces observations présente une grande cohérence dans la mesure où lorsque le TPA induit une diminution de $\mathrm{pH}$, il induit également une inhibition du trophisme et de la multiplication cellulaire (et vice versa). Les expériences primitivement réalisées avec le TPA ont été répétées avec des dérivés perméants du DG et des résultats identiques ont été obtenus. Il semble donc bien que le TPA activant la protéine kinase $C$.

Des exposés très complets concernant les oncogènes ont été faits dans le numéro inaugural de cette revue, rappelant l'importance des oncogènes en biologie. Il semble que divers produits d'oncogènes puissent agir à des niveaux différents dans la régulation du métabolisme des phosphoinositides. Les produits, pp6o ${ }^{\mathrm{v}-\mathrm{src}}$ et $\mathrm{p} 68^{\mathrm{v}-\mathrm{ros}}$, des oncogènes src du virus du sarcome de Rous et ros du virus du sarcome aviaire UR2 ont été primitivement décrits comme des kinases phosphorylant des polypeptides sur des résidus tyrosyl. Deux groupes de recherche indépendants ont montré que ces deux tyrosine kinases possédaient des activités phosphatidylinositol kinase et phosphatidylinositol-4phosphate kinase $[20,21]$.

\section{Phosphoinositides et oncogènes}

Un autre polypeptide, p2 ${ }^{\text {ras }}$, produit de l'oncogène ras, de poids moléculaire 21000 , pourrait également être impliqué dans la régulation de la voie des phosphoinositides. En effet, p2 ${ }^{\text {ras }}$ lie le GTP et peut l'hydrolyser [22, 23], propriété que possède également $\mathrm{Gs}$ du système adénylate cyclase. Il a été récemment montré que p2 ${ }^{\text {ras }}$ avait un effet sur l'activite de l'adénylate cyclase [24]. La similitude exposée plus haut entre la régulation de la synthèse de l'AMPc et celle du phosphatidylinositol - 4,5 - bisphosphate, rend vraisemblable l'hypothèse selon laquelle p2 $1^{\text {ras }}$ pourrait intervenir dans le métabolisme des phosphoinositides.

Le métabolisme des phosphoinositides joue donc un rôle primordial dans la régulation de nombreuses fonctions cellulaires. Le dérèglement de ce métabolisme pourrait être à l'origine de l'action de certains produits d'oncogènes. $\mathrm{L}^{\prime} \mathrm{IP}_{3}$ et le DG sont les seconds messagers, éléments essentiels de la transduction du message hormonal par cette voie métabolique. Deux publications récentes $[25,26]$ montrent que lè $\mathrm{DG}$ et l'IP $_{3}$ pourraient agir directement sur des éléments du cytosquelette. Ainsi le champ d'action de ces deux seconds messagers de l'action hormonale s'élargit encore...

\section{Summary}

The metabolism of phosphoinositides was recently shown to generate intracellular messengers as part of the hormonal signal transduction process. Phosphatidylinositol-4,5-bisphosdiacylglycerol and inositol1,4,5-trisphosphate. These two second messengers are involved in many cellular processes including cell growth and division, secretion, phototransduction. phate is hydrolysed into sn-1,2-

\section{TIRES A PART}

Ch. Le Peuch, U 249 de l'Inserm et I.P $8+02$ du Cnrs. BP 5051, 34033 Montpellicr. 